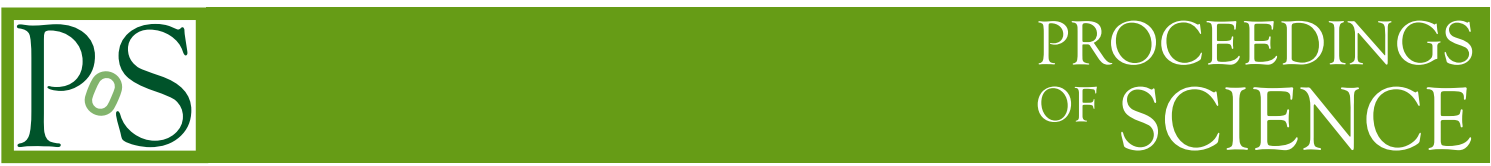

\title{
Triggering in ATLAS in Run 2 and Run 3
}

\section{Takuya Nobe* on behalf of the ATLAS collaboration}

International Center for Elementary Particle Physics (ICEPP), the University of Tokyo 7-3-1 Hongo, Bunkyo-ku, Tokyo 113-0033 Faculty of Science 10F, Japan

E-mail: Takuya.Nobe@cern.ch

The ATLAS experiment at the LHC records about $1 \mathrm{kHz}$ of physics collisions, out of the LHC design bunch crossing rate of $40 \mathrm{MHz}$. To achieve a high selection efficiency for physics events while reducing the significant background rate, a two-level trigger system is used. The event selection is based on physics signatures, such as the presence of energetic leptons, photons, jets or missing energy. In addition, the trigger system can exploit algorithms using topological information and multivariate methods to carry out the filtering for the many physics analyses pursued by the ATLAS collaboration. In Run 2, around 1500 individual selection paths (trigger chains) were used, with specified rate and the output bandwidth assignments. The optimization of the trigger menus (the lists of the trigger chains enabled during data taking) was performed using a dedicated framework running over $p p$ collisions data. The optimization of the Run-3 trigger menus is currently ongoing. For Run 3, the HLT software will be fully upgraded with a multithreading technique. Some improvements on the detector and electronics will be propagated to the trigger system. In this article, for example, the upgrade of the electron trigger project is presented briefly.

The Ninth Annual Conference on Large Hadron Collider Physics - LHCP2021

7-12 June 2021

Online

${ }^{*}$ Speaker 


\section{Introduction}

The successful operation of the ATLAS [1] trigger DAQ system in the LHC Run 2 in 2015-18 has led to fruitful physics results, such as measurements of the Standard Model processes in wide energy ranges, including a first measurement of the third-generation Yukawa coupling constants and more precise measurements of the $W / Z$ bosons and top quark. In addition there was a lot of progresses in searches for heavy new particles at the TeV scale and some lower- $p_{\mathrm{T}}$ processes, e.g. rare decays of $B$ hadrons. Currently, several upgrade projects are ongoing to make it possible that the Run 3 from 2022 and later continuously yield valuable physics results. In this article, some examples are presented of new features of the ATLAS trigger DAQ system introduced during Run 2 and those expected in Run 3.

\section{ATLAS trigger DAQ system}

The ATLAS trigger system is based on the physics “objects", such as high- $p_{\mathrm{T}}$ leptons, hadron jets, missing transverse energy $\left(E_{\mathrm{T}}^{\mathrm{miss}}\right)$ and so on. The LHC $p p$ collision events produced at $40 \mathrm{MHz}$ are first selected by Level-1 (L1) trigger at about $100 \mathrm{kHz}$ using custom made hardwares with a coarse granularity and lower energy/momentum resolutions. The software-based High-Level Trigger (HLT) follows and applies a more sophisticated selection to reduce the rate to around $1 \mathrm{kHz}$. Very tight event selections are necessary during data taking, to record target physics-event candidates with high efficiencies within the allowed data-taking rate. Since only the events selected by the trigger system are recorded, it is one of the most important parts for the ATLAS physics program. To fulfill the requirements for the rich physics program of ATLAS, events are selected in parallel by about 1500 "trigger chains". The chains are defined by the L1 decisions followed by a series of relevant HLT algorithms. For example, a "single muon trigger" requires at least one muon candidate at $\mathrm{L} 1$ with $p_{\mathrm{T}}>20 \mathrm{GeV}$, and at least one muon candidate at the HLT with $p_{\mathrm{T}}>26 \mathrm{GeV}$. It was used to take muons from $W / Z$ decays efficiently, with the recording rate about $200 \mathrm{~Hz}$ in Run 2 at peak instantaneous luminosity. The trigger system can exploit algorithms using more complex topological information and multivariate methods. For example, a " $B$-physics trigger" was defined which required at least one dimuon candidate with an invariant mass between 2 and $9 \mathrm{GeV}$ at L1, and 4 and $8.5 \mathrm{GeV}$ at the HLT with a better mass resolution. The L1Topo processor [2] newly introduced in Run 2 played an important role for these chains. In addition, there are some trigger-object level analysis chains, which records only HLT-level objects instead of the full detector information, the large reduction in data volume means that these data were recorded at a significantly larger rate of up to $10 \mathrm{kHz}[3,4]$. This made it possible to perform physics analyses at the lower- $p_{\mathrm{T}}$ range than otherwise allowed by baseline trigger chains. The "trigger menus" are defined by a list of chains enabled in the data taking, to achieve the total recording rate to be about $1 \mathrm{kHz}$. The enabling, disabling and prescaling of the individual trigger chains within a given menu can happen while a run in progress. This is used to match the active chains to the current instantaneous luminosity.

\section{Trigger menu development}

Physics results can be maximized by the optimization of the trigger menu to efficiently use all available resources. The development of the Run- 2 menus was aided by the use of the cost 
and rate estimation framework described in Ref. [5]. In this framework, trigger rate, CPU usage and the data-flow over DAQ network can be estimated using $p p$ data. It is achieved by rerunning the trigger algorithms on a special "enhanced-bias" dataset collected by several L1 trigger items from low- to high- $p_{\mathrm{T}}$. The prescales used to collect enhanced-bias data are invertible, for each event a weight is applied when the data are later used. This weight corrects for the effects of the online prescales and returns an effective zero-bias sample. The expected rates for individual trigger chains were estimated by this framework. It was confirmed they agree with the recorded rates to within the expected statistical precision for the majority of trigger chains. The menu optimization for Run 3 is currently ongoing. The estimated event rate and cost are extrapolated to the Run3 luminosity, assuming a linear dependency on instantaneous luminosity combined with ad-hoc scaling in center-of-mass energy.

\section{New features introduced in Run 2}

The rate of a $E_{\mathrm{T}}^{\text {miss }}$ trigger showed a non linear behavior with respect to the luminosity at the beginning of Run 2, as shown in Fig. 1a, due to a strong pileup dependency [6]. An initial calorimeter-cell-based $E_{\mathrm{T}}^{\text {miss }}$ reconstruction algorithm was replaced by a jet-based one, but the strong pileup dependency persisted. Finally a new pileup mitigation technique, so-called "pufit", was introduced, which corrects for pileup effects on high- $E_{\mathrm{T}}$ calorimeter signals contributing to $E_{\mathrm{T}}^{\text {miss }}$. The correction factor is obtained from a fit to lower- $E_{\mathrm{T}}$ signals. The pileup dependency was suppressed dramatically, as shown by the 2018 results in the figure.

Fig. 1b shows an advantage of the L1Topo processor. It shows an invariant mass distribution for the events taken by the standard dimuon chain and a $B$-physics chain using L1Topo and requiring a mass-window cut at $\mathrm{L} 1$ from 2 to $9 \mathrm{GeV}$ and a cut on $\Delta R_{\mu \mu}$ from 0.2 to 1.5 [2]. There was no bias on the shape introduced by the topological cuts at L1. The efficiency for the target signal events was kept to be about $80 \%$, while the L1 rate was significantly reduced by a factor of four.

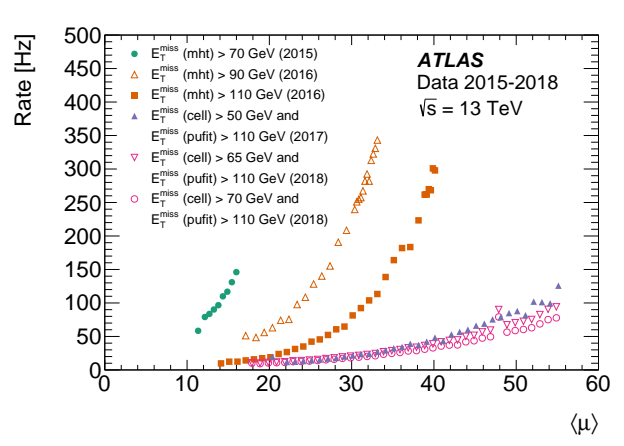

(a)

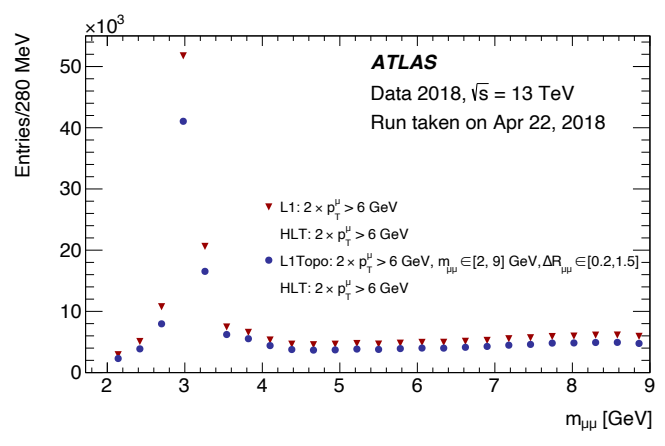

(b)

Figure 1: (a) HLT output rates, as a function of the average number of interaction per bunch crossing, $\langle\mu\rangle$, shown separately for example runs in each year, for triggers $E_{\mathrm{T}}^{\text {miss }}>70 \mathrm{GeV}$ (2015; cell based), $90 \mathrm{GeV}$ and $110 \mathrm{GeV}$ (2016; jet based), $E_{\mathrm{T}}^{\text {miss }}>110 \mathrm{GeV}$ (2017; with pufit pileup mitigation) and $E_{\mathrm{T}}^{\text {miss }}>110 \mathrm{GeV}$ (2018; a combination of pufit and cell-based algorithms $E_{\mathrm{T}}^{\text {miss }}>65 \mathrm{GeV}$ or $70 \mathrm{GeV}$ ) [6]. (b) Comparison of L1 dimuon triggers for muons with $p_{\mathrm{T}}>6 \mathrm{GeV}$ with and without topological requirements at L1 [2]. Invariant mass spectrum of the offline dimuon pair is shown for events selected by each trigger chain. 


\section{New features for Run 3}

The ATLAS HLT system exploits a multi-process forking approach to reduce the memory use. For Run 3, to better match the increasing CPU core count and the corresponding decreasing available memory per core, HLT software will be fully upgraded with the multithreading technique $[7,8]$.

Several upgrades of the detector and electronics are scheduled for Run 3. They will be propagated to the trigger system for better performances. For example, the LAr calorimeter readout will be upgraded to achieve finer granularity. New Feature EXtrapolator (FEX) processors will be installed to reconstruct electrons and jets more precisely at L1. Thanks to the higher granularity of the calorimeter read-out cells, shower shape information can be used to further reduce the background. For the electron triggers, using eFEX, an isolation requirement in $\eta$ direction and a cut on the energy ratio between electromagnetic and hadron calorimeter layers will be added to reduce the hadronic background. As shown in Fig. 2, a sharper turn-on curve can be achieved. The rate is reduced by a factor of two compared with the Run- 2 algorithm, which makes it possible to lower the electron- $E_{\mathrm{T}}$ threshold from $24 \mathrm{GeV}$ (in Run 2) to $21 \mathrm{GeV}$ at the same rate.

Many other improvements are expected, such as the installation of a new muon detector to reduce fake muon background [10] and HLT algorithms using machine learning techniques more extensively [11].

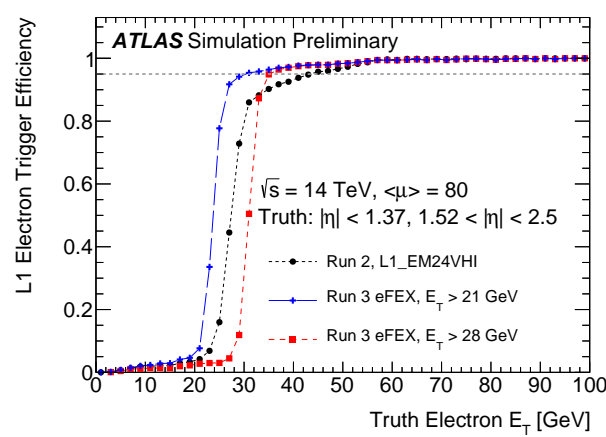

Figure 2: Trigger efficiency computed from a $Z \rightarrow e e$ simulation comparing the performance of the existing electron trigger with the proposed trigger that will be implemented for Run 3 [9]. The threshold of $21 \mathrm{GeV}$ (blue) has been chosen such that the trigger has about the same rate as the Run 2 baseline single electron trigger L1_EM24VHI (black). The threshold of $28 \mathrm{GeV}$ (red) results in about half that rate.

\section{Summary}

The ATLAS trigger and DAQ system in Run 2 and Run 3 data taking have been presented. In Run 2, around 1500 individual selection paths, the trigger chains, were used for data taking, each with specified rate and the output bandwidth assignments. The trigger menus, lists of the trigger chains, were optimized by a dedicated rate and cost estimation framework, using $p p$ collisions data. The optimization of the Run-3 menus are currently ongoing. For Run 3, the HLT software will be fully upgraded to utilize multithreading. Some improvements on the detector and electronics will be propagated to the trigger system. In this article, for example, the upgrade of the L1 electron trigger project has been reviewed briefly. 


\section{References}

[1] ATLAS Collaboration, The ATLAS Experiment at the CERN Large Hadron Collider, JINST 3 (2008) S08003.

[2] ATLAS Collaboration, Performance of the ATLAS Level-1 topological trigger in Run 2, submitted to EPJC, 2021. arXiv:2105.01416

[3] ATLAS Collaboration, Trigger-object Level Analysis with the ATLAS detector at the Large Hadron Collider: summary and perspectives, ATL-DAQ-PUB-2017-003.

[4] ATLAS Collaboration, Trigger Operation Public Results web page. http://twiki.cern. ch/twiki/bin/view/AtlasPublic/TriggerOperationPublicResults

[5] ATLAS Collaboration, Operation of the ATLAS trigger system in Run 2, JINST 15 (2020) P10004.

[6] ATLAS Collaboration, Performance of the missing transverse momentum triggers for the ATLAS detector during Run-2 data taking, JHEP 08 (2020) 80.

[7] G. A. Stewart, et. al., Multi-threaded software framework development for the ATLAS experiment, J. Phys.: Conf. Ser. 762 (2016) 012024.

[8] R. Bielski, ATLAS High Level Trigger within the multi-threaded software framework AthenaMT, J. Phys.: Conf. Ser. 1525 (2020) 012031.

[9] ATLAS Collaboration, Level-1 Calorimeter Trigger Public Results web page. https:// twiki.cern.ch/twiki/bin/view/AtlasPublic/L1CaloTriggerPublicResults

[10] ATLAS Collaboration, L1 Muon Trigger Public Results web page. https://twiki . cern. ch/twiki/bin/view/AtlasPublic/L1MuonTriggerPublicResults

[11] ATLAS Collaboration, HLT Tracking Public Results web page. https://twiki .cern.ch/ twiki/bin/view/AtlasPublic/HLTTrackingPublicResults 